\title{
The Effects of High and Low-Dose Cordyceps Militaris-Containing Mushroom Blend Supplementation After Seven and Twenty-Eight Days
}

\author{
Wesley David Dudgeon ${ }^{1,}$, , Dennison David Thomas ${ }^{1}$, William Dauch ${ }^{1}$, Timothy Paul Scheett ${ }^{1}$, \\ Michael John Webster ${ }^{2}$ \\ ${ }^{1}$ Department of Health and Human Performance, College of Charleston, Charleston, United States \\ ${ }^{2}$ School of Health Sciences, Valdosta State University, Valdosta, United States \\ Email address: \\ dudgeonw@cofc.edu (W. D. Dudgeon),ddthomas@cofc.edu (D. D. Thomas),dauchwj@g.cofc.edu (W. Dauch), \\ timscheett@gmail.com (T. P. Scheett),mjwebster@valdosta.edu (M. J. Webster) \\ ${ }^{*}$ Corresponding author
}

\section{To cite this article:}

Wesley David Dudgeon, Dennison David Thomas, William Dauch, Timothy Paul Scheett, Michael John Webster. The Effects of High and Low-Dose Cordyceps Militaris-Containing Mushroom Blend Supplementation After Seven and Twenty-Eight Days. American Journal of Sports Science. Vol. 6, No. 1, 2018, pp. 1-7. doi: 10.11648/j.ajss.20180601.11

Received: November 30, 2017; Accepted: December 8, 2017; Published: January 12, 2017

\begin{abstract}
The purpose of this study was to examine the aerobic performance effect of 1) low-dose mushroom blend supplementation (1.0 - $2.0 \mathrm{~g}$ /day) over a prolonged time period of 28 days compared to a placebo and 2) a higher dose of $\mathrm{PeakO}_{2}(12 \mathrm{~g} /$ day $)$ for seven days compared to placebo supplementation. For Trial 1, 40 young adult (19-34 yrs) subjects met participation criteria and were randomized into one of two groups. The treatment group $(\mathrm{T}, \mathrm{n}=23)$ consumed 1.0-2.0 g/day of mushroom blend $\left(\mathrm{PeakO}_{2}\right)$ along with $2.0 \mathrm{~g}$ of Gatorade powder for 28 days. The control group $(\mathrm{C}, \mathrm{n}=17)$ consumed placebo (whole wheat flour) and Gatorade powder in identical fashion. At baseline each participant completed a maximal oxygen consumption $\left(\mathrm{VO}_{2 \max }\right)$ test, which included a 5-minute economy state from minutes 3-8 along with a Wingate cycle ergometer test (peak power) at least $24 \mathrm{hrs}$ later. Forty-three young adult subjects met participation criteria for Trial 2 and were randomized into one of two groups. $\mathrm{T}(\mathrm{n}=29)$, consumed $12.0 \mathrm{~g}$ /day of mushroom blend $\left(\mathrm{PeakO}_{2}\right)$ along with $12.0 \mathrm{~g}$ of Gatorade powder for one week. C ( $\mathrm{n}=14)$ consumed placebo (whole wheat flour) and Gatorade powder in identical fashion There were no differences between groups in any variables at baseline. After 28 days of supplementation, $\mathrm{T}$ had a significant increase $(<0.05)$ in time to fatigue, a significant increase in $\mathrm{VO}_{2 \text { peak }}(\mathrm{p}<0.05)$ and a reduction in blood lactate $(\mathrm{p}<0.05)$ during the economy phase. Analysis for trial 2 was conducted stratifying each group by $\mathrm{VO}_{2 \text { peak }}$ at baseline, in which the top $50 \%$ of each group was compared to the bottom $50 \%$ (Treatment top, MT, Treatment bottom, MB, Control top, CT, Control bottom, CB). The MB group experienced significant $(\mathrm{p}<0.05)$ increases in $\mathrm{VO}_{2 \text { peak. }}$. $\mathrm{MB}$ increased $\mathrm{VO}_{2 \max }$ significantly $(p<$ 0.05 ) while MT, CT, and CB did not change significantly. The MT group experienced a significant $3 \mathrm{bpm}$ drop in economy HR from pre- to post-testing $(\mathrm{p}<0.05)$. The PT demonstrated a significant $4.5 \%$ increase in peak power from pre- to post-testing $(\mathrm{p}<0.05)$. No other changes were detected. These data suggest that longer duration, lower dose, supplementation of $\mathrm{PeakO}_{2}$ appears to improve endurance performance in apparently healthy young adults. Further, short duration supplementation of higher doses of $\mathrm{PeakO}_{2}$ may improve performance, but differing effects may occur based upon fitness level.
\end{abstract}

Keywords: Exercise, Cordyceps, Mushroom, Lactate, $\mathrm{VO}_{2}$

\section{Introduction}

Fungi have been used in medicine for many years to treat various conditions. The species sinensis and militaris of the genus Cordyceps have been studied for their medicinal and performance effects. Recent work has investigated the performance effects of Cordyceps militaris as it may have higher bioactivity compared to sinensis [1,2].

The commercially available supplement $\mathrm{PeakO}_{2}$ is an 
organic combination of six Ayurvedic mushroom strains. The main ingredient of this mushroom blend is Cordyceps militaris, which is a fungus belonging to the family Clavicipitaceae. The blend's high bioactivity, in combination with high antioxidants content, including Vitamin $\mathrm{D}_{2}$, LErgothioneine and Beta Glucans, may reduce oxidative stress placed on the body during exercise $[3,4]$. The main constituents of $\mathrm{PeakO}_{2}$ (at manufacturer's recommended supplementation dose of 4.0 grams/day) have been suggested to improve exercise endurance and power [5]. $\mathrm{PeakO}_{2}$ driven improvements in exercise performance may be attributed to improved respiratory control, blood flow, tissue oxygenation, oxygen uptake, lactate clearance, and energy turnover.

The primary purpose of this study was to examine the aerobic performance effect of low-dose mushroom blend supplementation (1.0 - $2.0 \mathrm{~g}$ /day) over a prolonged time period of 28 days compared to a placebo. Secondly, the researchers aimed to identify the effects of a higher dose of $\mathrm{PeakO}_{2}(12$ g/day) for only seven days compared to placebo supplementation on peak oxygen uptake, peak power output, and time to fatigue in apparently healthy adults. Third, the researchers aimed to investigate the effect of $\mathrm{PeakO}_{2}$ on steady state exercise economy $\left(\mathrm{VO}_{2}\right.$, lactate, and heart rate $\left.(\mathrm{HR})\right)$.

\section{Methods}

\subsection{Participants}

Recreationally active adults (19-34 yr) volunteered to participate in the study. All participants were assessed for inclusion/exclusion criteria on their first visit to the Human Performance Laboratory. Participant Inclusion/exclusion criteria consisted of the following: maintained a regular diet and exercise routine during the study period; not suspected of the following: an increased risk of cardiovascular disease, pregnancy, allergies to mushrooms, the use of medication for water retention, or any other contraindicated medical conditions; agreed to abstain from exercise and alcohol for 24 hours prior to testing; and agreed to notify a member of the research staff if they missed a supplement dosing.

Forty-three subjects ( 21 men, 22 women) met participation criteria for Trial 1 while another group of forty-three subjects participated in Trial 2. Forty subjects ( 24 men, 16 women) completed the trial 2 and their data were used in the final analysis. Of the three subjects who did not complete the full experimental protocol; one subject suffered an adverse response of gastrointestinal distress and had to withdraw; one subject suffered from severe quadriceps DOMs during retest due to an intense workout the day before; one subject was noncompliant, and removed from the study.

Trial-1: $1-2 \mathrm{~g} /$ day for 28 days

Table 1. Values are mean \pm standard deviation (SD).

\begin{tabular}{llll}
\hline Group & Age (yr) & Height $(\mathbf{c m})$ & Body Mass (kg) \\
\hline Mushroom $(\mathrm{n}=29)$ & $22.07 \pm 3.0$ & $169.7 \pm 9.0$ & $69.54 \pm 12.0$ \\
Placebo $(\mathrm{n}=14)$ & $21.86 \pm 2.3$ & $171 \pm 8.1$ & $71.0 \pm 11.5$ \\
\hline
\end{tabular}

Trial-2: $12 \mathrm{~g} /$ day for 7 days.
Table 2. Values are mean \pm standard deviation (SD).

\begin{tabular}{llll}
\hline Group & Age $(\mathbf{y r})$ & Height $(\mathbf{c m})$ & Body Mass $(\mathbf{k g})$ \\
\hline Mushroom $(\mathrm{n}=23)$ & $23.5 \pm 5.2$ & $172.9 \pm 8.8$ & $72.4 \pm 12.1$ \\
Placebo $(\mathrm{n}=17)$ & $22.5 \pm 4.6$ & $172.4 \pm 8.4$ & $72.3 \pm 11.8$ \\
\hline
\end{tabular}

\subsection{Methodology}

A randomized, placebo-controlled, single-blind design was used for both trials. All subjects signed a written informed consent approved by the College Institutional Review Board and completed a health history questionnaire and the Physical Activity Readiness Questionnaire (PAR-Q). Following clearance for participation, all subjects completed baseline testing of anthropometric measurements and oxygen consumption $\left(\mathrm{VO}_{2 \text { peak }}\right)$. Additionally, participants in Trial 2 also completed a Wingate anaerobic power test at least 24 hours following $\mathrm{VO}_{2 \text { peak }}$ testing.

In Trial-1, participants were randomly assigned to one of two supplementation groups, 1-2 g/day of mushroom blend or $2 \mathrm{~g}$ /day of a color-matched placebo consisting of wholewheat flour. Participants then proceeded with daily supplementation for 28 days before returning to the laboratory for follow-up testing in the same manner as baseline testing. In Trial-2, participants were randomly assigned to one of two groups, $12 \mathrm{~g} /$ day of mushroom blend or $12 \mathrm{~g}$ /day of the placebo control. Following seven days of supplementation, participants returned for a follow-up $\mathrm{VO}_{2 \text { peak }}$ test. After a total of eight days of supplementation the Wingate anaerobic power test was repeated to allow for the same 24-hour rest interval and to include any potential acute $(<24 \mathrm{~h})$ supplementation effects. In both trials, a matching dose of Gatorade ${ }^{\circledR}$ Thirst Quencher Powder Orange was added for flavor to all supplements and placebos.

\subsubsection{Peak Oxygen Consumption Testing}

All subjects performed a $\mathrm{VO}_{2 \text { peak }}$ test on a Monark electrically-braked cycle ergometer (Monark Ergomedic 894E, Varberg, Sweden). Following anthropometric assessment, each subject was fitted on the cycle ergometer, with the handlebars adjusted to a comfortable setting, and the seat height selected to allow no more than approximately $15^{\circ}$ flexion at the knee during extension. Heart rate was measured throughout the test using a Polar H7 heart rate monitor (Polar, Lake Success, NY). Subjects were instructed to pedal at a cadence of 50 revolutions $/ \mathrm{min}$ for the duration of the test. During the first stage of the test, subjects pedaled for two minutes at a power output of 50 watts. Stage 1 was followed by a 5-minute economy phase at a workload of 75 watts and 100 watts for females and males, respectively. During this stage, HR, blood lactate, and $\mathrm{VO}_{2}$ data were collected. Blood lactate (Lactate Plus, Nova Biomedical, Waltham, MA) samples were collected via finger capillary puncture at the $5^{\text {th }}$ minute $(3$ minutes into the $2^{\text {nd }}$ stage) of the test. Each successive stage (stages 3 through termination) of the test was realized by a workload increase of 25 watts and 50 watts for females and males, respectively. At the end of each stage subjects were 
asked to indicate their rating of perceived exertion (RPE) using a 6-20 rating scale (original Borg scale). The test was terminated when the subject could no longer maintain 50 revolutions/min, or until volitional fatigue. Time to fatigue was recorded at the end of the test. $\mathrm{VO}_{2 \text { peak }}$ was determined by respiratory gas analysis which was measured continuously throughout the test (Parvo Medics True Peak 2400, Salt Lake City, UT).

\subsubsection{Wingate Anaerobic Power Test}

Following a period of at least $24 \mathrm{~h}$, subjects returned to the Human Performance Laboratory to complete a Wingate Anaerobic Power test. Upon arrival, a finger capillary blood sample was collected in a microcuvette with reagents and analyzed for hemoglobin and hematocrit (Alere, HemoPoint H2, Germany). For each subject, the cycle ergometer seat and handle bar positioning specifications were set to those used during the $\mathrm{VO}_{2 \text { peak }}$ test the preceding day. Prior to the test, each subject completed a 5-minute warm-up, which consisted of pedaling at 50 revolutions/min (50 watts) for 55 seconds, followed by a maximal 5 -second sprint, after which the subject returned to pedaling at 50 revolutions/min. After completing the warm-up the subject rested for 2-5 minutes (standing or walking). After returning to the cycle, $1 / 3$ of the workload being used for the test was applied ( $2.5 \%$ of body mass), with the subject pedaling slowly for 10 seconds. The resistance was then removed, the subject was instructed to pedal maximally, and after $\sim 2 \mathrm{~s}$ the target resistance $(7.5 \%$ of the subject's body mass) was applied to the flywheel and the subject continued to pedal maximally for $30 \mathrm{~s}$. The resistance was then immediately removed and the subject completed a 2-3minute cool down during which they pedaled freely against a resistance of $1.0 \mathrm{~kg}$. Peak power output was recorded and calculated by Monark ATS software (Monark, Varberg, Sweden), specific for Monark Ergomedic 894E Wingate tests.

\subsubsection{Statistical Analysis}

All normally distributed data were analyzed using Sigma Sat v3.5 (Point Richmond, CA) or Microsoft Excel (Redmond, WA). Dependent measures t-tests were used to evaluate changes within mushroom groups and independent measures t-tests were used to compare differences between groups. All data are presented as mean \pm standard deviation (SD).

\section{Results}

\subsection{Trial 1}

\subsubsection{Time to Fatigue}

Following 28 days of supplementation, the mushroom group showed a significant pre-post improvement in time to fatigue during the test for $\mathrm{VO}_{2 \text { peak, }}$, whereas the control group demonstrated no change (Mushroom: $25.8 \pm 61.1 \mathrm{sec}, \mathrm{p}<$ 0.05; Control: $0.5 \pm 30.5 \mathrm{sec}, \mathrm{p}>0.05)$. The change in the mushroom group showed a trend $(\mathrm{p}<0.07)$ for difference from the change in the control group.

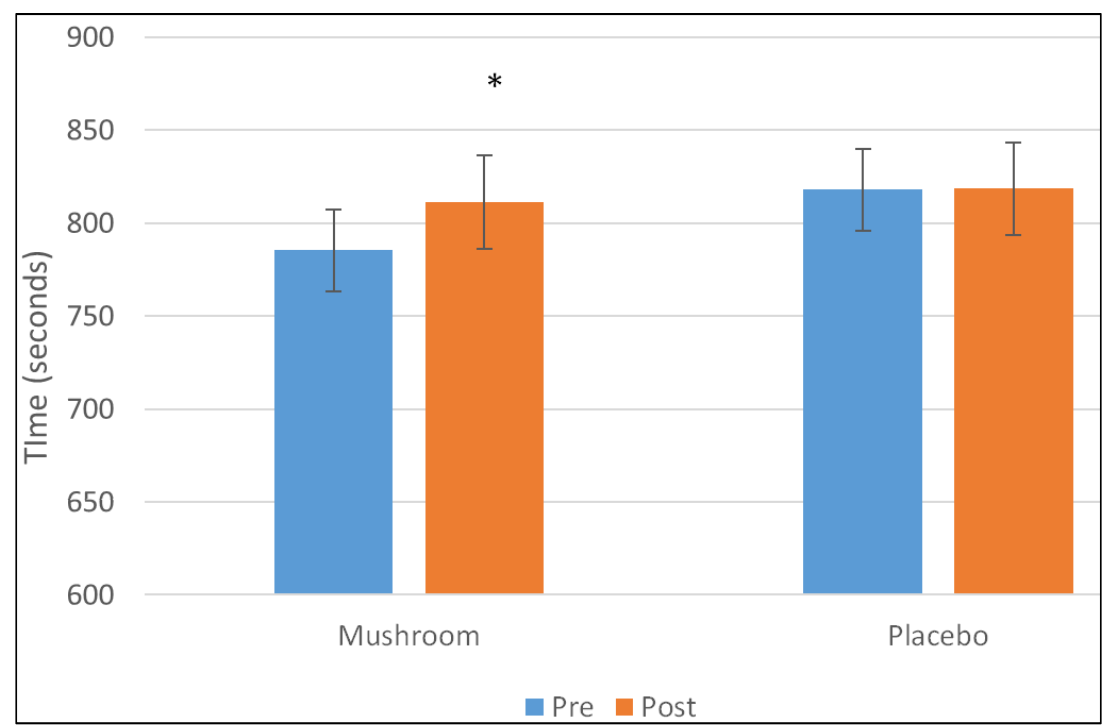

Figure 1. Changes in time to fatigue from pre to post supplementation (28 days). Values are mean \pm standard error. *indicates within group difference at $P<0.05$.

\subsection{2. $\mathrm{VO}_{2 p e a k}$}

Table 3. Values are mean \pm standard deviation (SD).

\begin{tabular}{|c|c|c|c|c|}
\hline Group & Baseline $\mathrm{VO}_{2 \text { peak }}(\mathrm{mL} / \mathrm{kg} / \mathrm{min})$ & Post $\mathrm{VO}_{\text {2peak }}(\mathrm{mL} / \mathrm{kg} / \mathrm{min})$ & Change (mL/kg/min) & t-Test \\
\hline Mushroom & $40.1 \pm 6.1$ & $41.1 \pm 6.2^{*}$ & $1.0 \pm 2.3$ & $p=0.02$ \\
\hline Placebo & $41.7 \pm 6.1$ & $42.5 \pm 6.0$ & $0.8 \pm 2.1$ & $p=0.09$ \\
\hline
\end{tabular}


The mushroom group demonstrated a significant increase in $\mathrm{VO}_{2 \text { peak }}(\mathrm{p}<0.05)$ while the control group did not exhibit any changes in $\mathrm{VO}_{2 \text { peak }}$ (Table 3)

\subsubsection{Blood Lactate}

Measured during stage 2 (at a workload of 75 watts for females and 100 watts for males) of the test for $\mathrm{VO}_{2 \text { peak }}$, blood lactate significantly decreased $(0.22 \pm 0.41 \mathrm{mM}, \mathrm{p}<$ $0.05)$ in the mushroom group whereas the control group demonstrated no change $(0.01 \pm 0.52 \mathrm{mM}, \mathrm{p}>0.05)$. The change in the mushroom group was different $(\mathrm{p}<0.05)$ than the change in the control group.

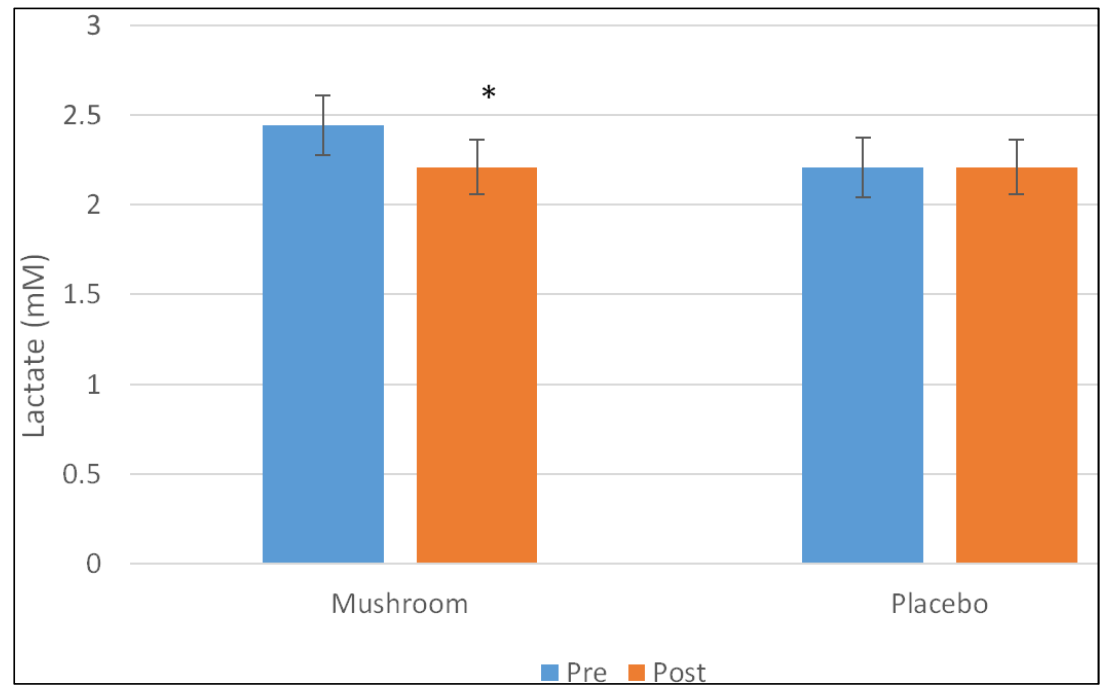

Figure 2. Changes in submaximal blood lactate concentration from pre to post supplementation (28 days). Values are mean \pm standard error.

\subsection{Trial 2}

During trial 2, initial analysis revealed no significant changes for any variables measured. Post-hoc analysis was conducted by stratifying each group at the $50^{\text {th }}$ percentile by aerobic capacity as measured during initial testing for $\mathrm{VO}_{2 \text { peak. }}$ This resulted in the following groups: mushroom bottom group (MB), $\mathrm{N}=11$, mushroom top group (MT), $\mathrm{N}=$ 12), placebo bottom (PB), $N=8$ and placebo top (PT), $N=9$.

\subsection{1. $\mathrm{VO}_{2 P e a k}$}

The MB group experienced significant $(\mathrm{p}<0.05)$ increases in $\mathrm{VO}_{2}$ peak from pre- to post-testing, whereas the MT group did not significantly differ in $\mathrm{VO}_{2 \text { peak }}$ from pre- to post-testing. Neither PT nor PB groups demonstrated significant changes in $\mathrm{VO}_{2 \text { peak }}$ from pre- to post-testing. $\mathrm{MB}$ changes in $\mathrm{VO}_{2}{ }^{\text {peak }}$ from pre- to post-testing were significantly greater than the MT group (MB $1.95 \pm 2.7$, MT $0.44 \pm 2.9 \mathrm{ml} / \mathrm{kg} / \mathrm{min} \mathrm{p}<0.05$ ).

Table 4. Values are mean \pm standard deviation (SD).

\begin{tabular}{llll}
\hline & Mushroom Top (MT) & Mushroom Bottom (MB) & Placebo Top (PT) \\
\hline Pre $\mathrm{VO}_{2 \text { peak }}(\mathrm{ml} / \mathrm{kg} / \mathrm{min})$ & $45.4 \pm 3.8$ & $36.9 \pm 3.8$ & $46.8 \pm 3.0$ \\
${\text { Post } \mathrm{VO}_{2 \text { peak }}(\mathrm{ml} / \mathrm{kg} / \mathrm{min})}^{4}$ & $44.9 \pm 4.2$ & $38.9 \pm 4.4^{*}$ & $47.3 \pm 3.0$ \\
\hline
\end{tabular}

*indicates within group difference at $\mathrm{P}<0.05$.

\subsubsection{Time to Fatigue}

Both the MT group and the PT group had significant $(\mathrm{p}<0.05)$ increases in time to fatigue (TTF) from pre- to post-testing $(2.7 \%$ and $2.9 \%$, respectively). There was no significant difference $(\mathrm{p}>0.05)$ in TTF change between the TT and PT groups. TTF did not change from pre- to post-testing in the MB group or the PB group.

Table 5. Values are mean \pm standard deviation (SD).

\begin{tabular}{llll}
\hline & Mushroom Top (MT) & Mushroom Bottom (MB) & Placebo Top (PT) \\
\hline Pre Time to Fatigue (sec) & $829.4 \pm 118.2$ & $785.5 \pm 115.3$ & $845.2 \pm 95.3$ \\
Post Time to Fatigue (sec) & $852.6 \pm 116.5 *$ & $787.5 \pm 97.7$ & $870.7 \pm 100.5 *$ \\
\hline
\end{tabular}

*indicates within group difference at $\mathrm{p}<0.05$.

\subsubsection{Peak Power Output}

The PT demonstrated a significant $4.5 \%$ increase in peak power from pre- to post-testing $(\mathrm{p}<0.05)$. The MT and MB group's absolute PPOs did not change significantly from pre- to post-testing. The PB group's absolute PPOs did not change significantly from pre- to post-testing. However, the MB of the mushroom group had a $2.6 \%$ increase in absolute PPO from 
pre- to post-testing and was trending towards significance $(\mathrm{p}=0.056)$.

Table 6. Values are mean \pm standard deviation (SD).

\begin{tabular}{lllll}
\hline & Mushroom Top (MT) & Mushroom Bottom (MB) & Placebo Top (PT) & Placebo Bottom (PB) \\
\hline Pre Peak Power (watts) & $932.7 \pm 235.5$ & $919.9 \pm 238.9$ & $907.5 \pm 181.5$ & $945.3 \pm 253.9$ \\
Post Peak Power (watts) & $947.9 \pm 197.0$ & $944.6 \pm 224.6$ & $949.7 \pm 209.4^{*}$ & $966.5 \pm 285.4$ \\
\hline
\end{tabular}

*indicates within group difference at $\mathrm{p}<0.05$.

\subsubsection{Submaximal Heart Rate}

The MT group experienced a significant $3 \mathrm{bpm}$ drop in economy HR from pre- to post-testing $(\mathrm{p}<0.05)$. There were no significant changes in economy HR from pre- to posttesting for the MB. There were no significant changes in economy HR from pre- to post-testing for the PT or the PB groups. However, changes in economy HR for the MT were not different compared to economy HR changes in the $\mathrm{MB}$ group $(p>0.05)$ or economy HR changes in the PT group $(\mathrm{p}>0.05)$.

Table 7. Values are mean \pm standard deviation (SD).

\begin{tabular}{lllll}
\hline & Mushroom Top (MT) & Mushroom Bottom (MB) & Placebo Top (PT) & Placebo Bottom (PB) \\
\hline Pre Heart Rate (BPM) & $134 \pm 19$ & $132 \pm 16$ & $129 \pm 12$ & $134 \pm 13$ \\
Post Heart Rate (BPM) & $131 \pm 19 *$ & $134 \pm 10$ & $127 \pm 8$ & $134 \pm 13$ \\
\hline
\end{tabular}

*indicates within group difference at $\mathrm{p}<0.05$.

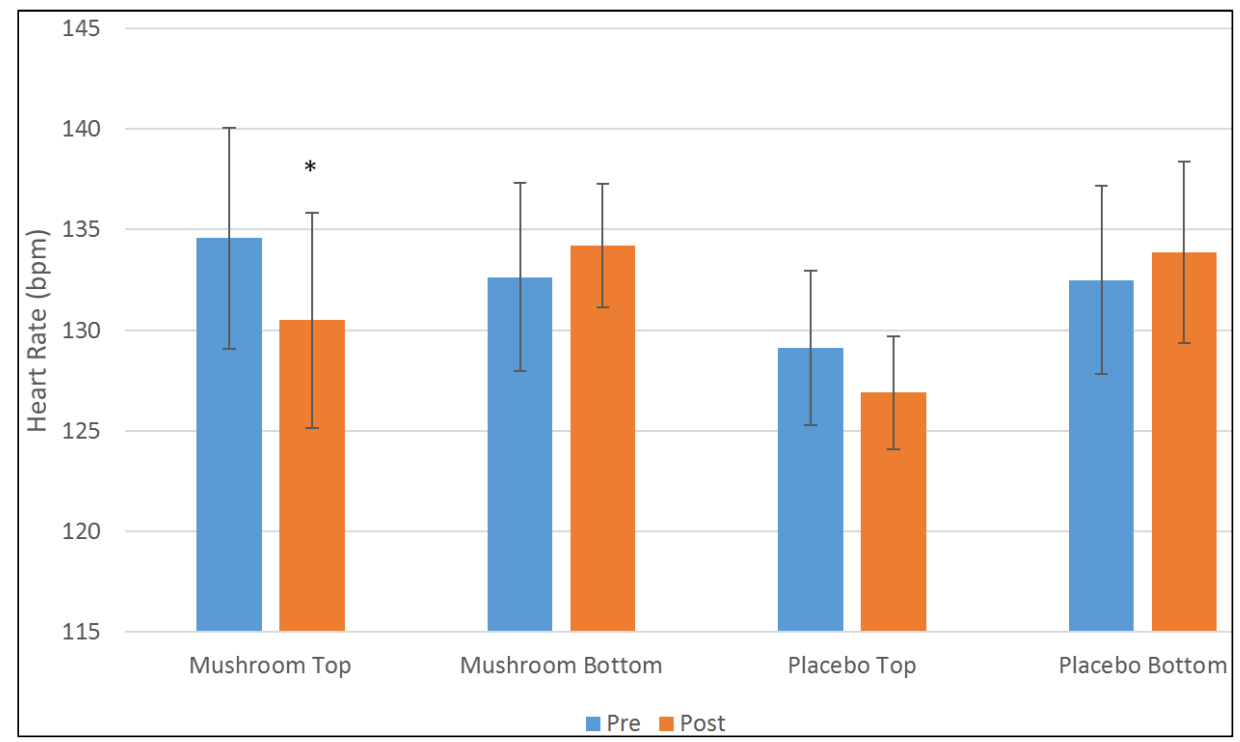

Figure 3. Changes in submaximal heart rate from pre to post supplementation (7 days). Values are mean \pm standard error.

\section{Discussion}

Very little work is reported in the literature investigating the impact of mushroom blends on exercise performance in humans. Thus, this study aimed to determine the impact of high and low-dose mushroom blend supplementation, over 7 and 28 days respectively, on various markers of exercise performance including $\mathrm{VO}_{2 \text { peak }}$, endurance, and submaximal stress. While not definitive, and warranting more investigation, these findings of improved time to fatigue, increased $\mathrm{VO}_{2 \text { peak }}$ and reduced submaximal lactate and $\mathrm{HR}$ suggest a role of $\mathrm{PEAKO}_{2}$ supplementation in improving markers of endurance exercise performance.

A recent investigation of mushroom supplementation and performance in apparently healthy young adults [5] evaluated a $4 \mathrm{~g}$ /day dose of $\mathrm{PEAKO}_{2}$ over 7- and 21-days, and indicated that only after 21 days of supplementations were benefits realized. Cordyceps militaris supplementation in animal models has suggested dosages of up to $4 \mathrm{~g} / \mathrm{kg}$ to be safe (equivalent of $\sim 300 \mathrm{~g} /$ day in a human population), while lower dosages (less than $2.5 \mathrm{~g}$ ) for shorter periods (less than 10 days) have not resulted in performance benefits in humans $[6,7]$ while large doses (4.5 g/day) have elicited performance benefits. Further complicating the picture are data demonstrating that $3 \mathrm{~g}$ /day for 35 days did not impact performance in fit cyclists [8]. However, it should be noted that the subjects referenced in these studies were of varying fitness levels, making interpretation difficult.

Seven days of dosing in C57-Bl/6 mice (both $200 \mathrm{mg} / \mathrm{kg}$ and $400 \mathrm{mg} / \mathrm{kg}$ ) demonstrated a higher hepatic bioenergy status (increased ATP) [9]. Twenty-eight days of cordyceps sinesis supplementation $(100 \mathrm{mg} / \mathrm{kg}, 200 \mathrm{mg} / \mathrm{kg}$ and 400 $\mathrm{mg} / \mathrm{kg}$ ) in mice demonstrated longer swimming durations, increase hepatic and muscle glycogen, and decreased blood lactate compared to controls [10], while 15 days of 
supplementation $(200 \mathrm{mg} / \mathrm{kg})$ increased antioxidant response and improved endurance in rats [11].

Work in humans has used dosages significantly lower than that tested on animals (only $\sim 5 \%$ of that used in animals), and results have been equivocal. Further, the manufacturer recommends consuming $4 \mathrm{~g}$ /day with no mention of duration, though evidence for this dose is not found in the literature. Thus, the goal was to determine if a larger dosage (12 g/day) for 7 days (less time than other published human work) would be impactful, while also testing a lower dose (1$2 \mathrm{~g} /$ day) while extending the dosing out to 28 days (both less mushroom and longer time) to see if this combination would have an impact.

This young adult, mixed gender, population (19-34 yr) with an average $\mathrm{VO}_{2 \text { peak }}$ of greater than $40 \mathrm{ml} / \mathrm{kg} / \mathrm{min}$ would be categorized as above average to good [12]. With a dose of 1-2 g/day over 28 days, the mushroom group exhibited a significant $26 \pm 61 \mathrm{sec}$ improvement in TTF while also showing a significant $2.5 \%$ increase in $\mathrm{VO}_{2 \text { peak }}$. While these improvements in oxygen consumption and time to fatigue are less than reported by Hirsch et al. 2016, the dosage used in the present study was also less (1-2g/day vs $4 \mathrm{~g}$ /day).

The finding of reduced blood lactate during steady state submaximal exercise in the mushroom group is novel and suggests an influence on substrate utilization, ATP production and/or oxygen kinetics.

Trial 1 utilized a longer duration lower dose protocol compared with previous reported work. While the exact mechanism for the benefits of $\mathrm{C}$. militaris on exercise performance is not known, both animal [13] and human [1416] studies suggest supplementation results in improved work output. Improved ATP:Pi [17], and/or more efficient oxygen utilization leading to improved exercise economy [18], antioxidant effects $[15,16,19]$ and inhibition of the HPA axis [20, 21] have all been posed as potential mechanisms of action.

The second arm of this study, looking at a higher dose (12 $\mathrm{g}$ /day) for seven days, also showed that the mushroom blend had an impact on performance. While no significant findings were observed when studying the mushroom group as a whole $(\mathrm{N}=23)$, other work has suggested a differing effect based on fitness $[8,14]$, so groups were stratified by $\mathrm{VO}_{2 \text { Peak, }}$, dividing them into groups above and below the median percentile. Those in the lower aerobic fitness group that supplemented (MB) increased $\mathrm{VO}_{2 \text { Peak }}$ and TTF, while those who were of higher aerobic fitness at study initiation (MT) also increased TTF. The MT group also exhibited a lower HR during steady state exercise following supplementation. However, this should be viewed cautiously as the change in the MT was not significantly different than the changes (no other groups had significant changes) in the other groups. Also, the PT did show an increase in TTF.

These findings are novel in that no previous human work have reported impacts after only 7 days of supplementation. Additionally, this work demonstrated that aerobic fitness level may influence the impact of the mushroom blend. This is not totally unexpected, given that those who are already fit have less room for improvements and would also tend to take longer time duration to realize benefits.

\section{Conclusion}

Taken together, these data show that $\mathrm{PEAKO}_{2}$ had a significant impact on markers of aerobic fitness in young, apparently healthy adults. A supplement that improves oxygen consumption, time to fatigue, blood lactate levels, or exercise HR, taken individually would be reason for encouragement in that it takes significant training time to change these variables independent of supplementation. Taken together, these findings suggest that Cordyceps militaris, administered in the blend provided by $\mathrm{PEAKO}_{2}$, can have significant impacts of endurance exercise performance. More work is needed to determine the optimal supplement dosage and duration needed to realize these benefits, and as yet no mechanisms for action have been fully elucidated.

\section{Acknowledgements}

This study was funded by Disruptive Nutrition, Burlington, NC.

\section{References}

[1] H. O. Kim and J. W. Yun, "A comparative study on the production of exopolysaccharides between two entomopathogenic fungi Cordyceps militaris and Cordyceps sinensis in submerged mycelial cultures," (in eng), J Appl Microbiol, vol. 99, no. 4, pp. 728-38, 2005.

[2] H. M. Yu, B. S. Wang, S. C. Huang, and P. D. Duh, "Comparison of protective effects between cultured Cordyceps militaris and natural Cordyceps sinensis against oxidative damage," (in eng), J Agric Food Chem, vol. 54, no. 8, pp. 3132-8, Apr 192006.

[3] J. J. Cannell, B. W. Hollis, M. B. Sorenson, T. N. Taft, and J. J. Anderson, "Athletic performance and vitamin D," (in eng), Med Sci Sports Exerc, vol. 41, no. 5, pp. 1102-10, May 2009.

[4] C. Xu, J. Lv, Y. M. Lo, S. W. Cui, X. Hu, and M. Fan, "Effects of oat beta-glucan on endurance exercise and its anti-fatigue properties in trained rats," (in eng), Carbohydr Polym, vol. 92, no. 2, pp. 1159-65, Feb 152013.

[5] K. R. Hirsch, A. E. Smith-Ryan, E. J. Roelofs, E. T. Trexler, and M. G. Mock, "Cordyceps militaris Improves Tolerance to High-Intensity Exercise After Acute and Chronic Supplementation," (in eng), J Diet Suppl, pp. 1-13, Jul 13 2016.

[6] S. N. Colson, F. B. Wyatt, D. L. Johnston, L. D. Autrey, Y. L. FitzGerald, and C. P. Earnest, "Cordyceps sinensis- and Rhodiola rosea-based supplementation in male cyclists and its effect on muscle tissue oxygen saturation," (in eng), $J$ Strength Cond Res, vol. 19, no. 2, pp. 358-63, May 2005.

[7] C. P. Earnest et al., "Effects of a commercial herbal-based formula on exercise performance in cyclists," (in eng), Med Sci Sports Exerc, vol. 36, no. 3, pp. 504-9, Mar 2004. 
[8] A. C. Parcell, J. M. Smith, S. S. Schulthies, J. W. Myrer, and G. Fellingham, "Cordyceps Sinensis (Cordy Max Cs-4) supplementation does not improve endurance exercise performance," (in eng), Int J Sport Nutr Exerc Metab, vol. 14, no. 2, pp. 236-42, Apr 2004.

[9] G. Dai, T. Bao, C. Xu, R. Cooper, and J. S. Zhu, "CordyMax Cs-4 improves steady-state bioenergy status in mouse liver," (in eng), J Altern Complement Med, vol. 7, no. 3, pp. 231-40, Jun 2001.

[10] F. Yan, Y. Zhang, and B. Wang, "Effects of polysaccharides from Cordyceps sinensis mycelium on physical fatigue in mice," Bangladesh Journal of Pharmacology, vol. 7, no. 3, pp. 217-221, 2012.

[11] R. Kumar, P. S. Negi, B. Singh, G. Ilavazhagan, K. Bhargava, and N. K. Sethy, "Cordyceps sinensis promotes exercise endurance capacity of rats by activating skeletal muscle metabolic regulators," (in eng), J Ethnopharmacol, vol. 136, no. 1, pp. 260-6, Jun 142011.

[12] A. C. o. S. Medicine, ACSM's Resource Manual For Guidelines for Exercise Testing and Prescription, 7 ed. Wolters Kluwer, 2013.

[13] K. Jung, I. H. Kim, and D. Han, "Effect of medicinal plant extracts on forced swimming capacity in mice," (in eng), $J$ Ethnopharmacol, vol. 93, no. 1, pp. 75-81, Jul 2004.

[14] C. Y. Chen et al., "Rhodiola crenulata- and Cordyceps sinensis-based supplement boosts aerobic exercise performance after short-term high altitude training," (in eng), High Alt Med Biol, vol. 15, no. 3, pp. 371-9, Sep 2014.
[15] Z. Zhang and e. al., "Clinical and laboratory studies of Jin Shui Bao in eliminating oxygen free radicals in elderly senescent Xu-Zheng (Asthenia syndrome) patients," Journal of Management of Traditional Chinese Medicine, vol. 5, pp. 14-18(supplement), 1995.

[16] Z. Cao and Y. Wen, "Therapeutic effect analysis of Jin Shui Bao capsule in treatment of 33 elderly Xu-Zheng (Asthenia syndrome) patients," Journal of Applied Traditional Chinese Medicine, vol. 1, pp. 32-33, 1993.

[17] N. Manabe et al., "Effects of the mycelial extract of cultured Cordyceps sinensis on in vivo hepatic energy metabolism in the mouse," (in eng), Jpn J Pharmacol, vol. 70, no. 1, pp. 858, Jan 1996.

[18] Y. Lou, X. Liao, and Y. Lu, "Cardiovascular pharmacological studies of ethanol extracts of Cordyceps mycelia and Cordyceps fermentation solution," Chinese Traditional and Herbal Drugs, vol. 17, no. 5, pp. 7-21, 1986.

[19] W. Wang, "Observations of effects of Jin Shui Bao on SOD activity in COED patients," Journal of Management of Traditional Chinese Medicine, vol. 5, p. 24(supplement), 1995.

[20] Y. Z. Guo, "Medicinal chemistry, pharmacology, and clinical applications of fermented mycelia of Cordyceps sinensis and JinShuBao capsule," Journal of Modern Diagnostics and Therapeutics, no. 1, pp. 60-65, 1986.

[21] Y. Huang et al., "Toxicity study of fermentation Cordyceps mycelia B414," Zhongchenyao Yanjiu, vol. 10, pp. 24-25, 1987. 